\section{La intervención social en clave de innovación social. Un análisis desde dos proyectos de extensión universitaria}

\author{
Marcos Exequiel Angeloni \\ Secretaría de Extensión Social y Cultural. \\ Universidad Nacional del Litoral, Argentina. \\ marcosangelonisj@gmail.com

\section{Julio César Lozeco} \\ Programa de Extensión de Economía Social \\ y Solidaria. Universidad Nacional del Litoral, \\ Argentina. \\ juliolozeco@gmail.com
}

\author{
Sofía Clarisa Marzioni \\ Consejo Nacional de Investigaciones \\ Científicas y Técnicas. Universidad \\ Nacional del Litoral, Argentina. \\ sofimarzioni@hotmail.com
}

\section{María Elena Kessler} de Extensión (2015-2018). Universidad
Dirección de Integración de la Extensión, la Investigación y las Políticas Públicas. Secretaría Nacional del Litoral, Argentina. mileva143@gmail.com

Social intervention in the key of social innovation. An analysis from two university extension projects

\section{Abstract}

The article analyzes, from two selected cases that are framed in extension projects of the Universidad Nacional del Litoral, the relationships that are generated between the extension teams (conformed by teachers-researchers, students and specialists) and the territorial actors involved in intervention processes. It is nourished by the different moments in the framework of the project Action Course for Research and Development (CAI + D, in spanish) entitled: "Analysis of social innovation from scientific and technological transfer between Universidad Nacional del Litoral and institutions of the public sphere" (2011). The reflection is focused on the network of relationships that are knotted, the senses, the brands that these associations produce, as innovative processes that try to be an answer to the problems that originate them.

Keywords: university extension, social innovation, scientific-technological transfer, public policies.
Investigación y extensión universitaria / Intervenciones
A intervenção social em chave de inovação social. Uma análise a partir de dois projetos de extensão universitária

\section{Resumo}

$\mathrm{O}$ artigo analisa, a partir de dois casos selecionados, no âmbito de projetos de extensão da Universidad Nacional del Litoral, as relações que são geradas entre as equipes de extensão (formadas por professorespesquisadores, estudantes e especialistas) e os atores territoriais envolvidos nos processos de intervenção. Nutre-se dos diferentes momentos do projeto Curso de Ação para a Pesquisa e o Desenvolvimento (CAI+D, em espanhol) intitulado: "Análise das inovações sociais, a partir da transferência científico-tecnológica, entre a Universidad Nacional del Litoral e instituições da esfera pública" (convite 2011). A reflexão se concentra na rede de relacionamentos que se amarram, os sentidos, as marcas que essas associações produzem, como processos inovadores que tentam ser uma resposta aos problemas que os originam.

Palavras-chave: extensão universitária, inovação social, transferência científicotecnológica, políticas públicas innovación social, transferencia científicotecnológica, políticas públicas. 


\section{Introducción}

La Universidad Nacional del Litoral (UNL) desarrolla, desde distintos dispositivos — los servicios altamente especializados a terceros, los Cursos de Acción para la Investigación y el Desarrollo (CAI+D) orientados a problemas sociales y productivos y los proyectos de extensión-, ${ }^{1}$ procesos de transferencia de conocimientos a diversos actores de la esfera pública (estatal y no estatal) y privada. La finalidad principal de los mismos es generar ámbitos de cooperación entre la academia y la sociedad que logren transformar las prácticas en el campo social, en las diferentes áreas en las que intervienen —salud, educación, medioambiente, género, economía social, poblaciones expuestas a situaciones de vulnerabilidad y riesgo-, compartiendo saberes, percepciones, aprendizajes, como un aporte también a procesos colectivos y de inclusión social.

En este trabajo nos proponemos analizar el entramado de relaciones que se establecen entre los integrantes de los equipos de extensión y actores sociales, ajenos al ámbito científico-académico, que son referentes de organizaciones de la sociedad civil y representantes de agencias gubernamentales. Estas reflexiones, a su vez, nos remiten al CAl+D "Análisis de las innovaciones sociales, a partir de la transferencia científico-tecnológica, entre la Universidad Nacional del Litoral e instituciones de la esfera pública" (convocatoria 2011).

La UNL dispone de un sistema de registro histórico de su práctica académica conformado por publicaciones, documentos y materiales audiovisuales. Sin embargo, es limitada la información en torno a la incidencia que las intervenciones generan con relación a un conjunto de cuestiones en el marco de las interacciones que promueven. Las mismas refieren a: identificación de los aprendizajes producto de las experiencias desarrolladas; intercambio de saberes y percepciones entre el equipo extensionista y los actores sociales; visibilización de prácticas novedosas, creativas; explicitación de los logros alcanzados como respuesta a la problemática que abordan; identificación de los entramados asociativos y, por último, articulación de las intervenciones con las políticas públicas como factor de sustentabilidad.

Para realizar el análisis que nos proponemos, seleccionamos dos casos a partir de los cuales reflexionamos en torno a los procesos que tienen lugar desde las intervenciones que los equipos extensionistas promueven, en su vinculación con los actores sociales, no académicos, pertenecientes al espacio público, y lo hacemos en clave de innovación social. Los mismos fueron elegidos desde las categorías que el equipo de investigación consensuó respecto de dicho concepto, al cual haremos referencia más adelante.

Este trabajo se organiza de la siguiente manera: en primer lugar, damos cuenta de cuatro cauces teóricos desde donde pensar la extensión universitaria crítica. En segundo lugar, compartimos el concepto de innovación social que acuñamos, producto de un recorrido teórico y discusiones en el seno del equipo. En tercer lugar, presentamos brevemente el CAl+D de referencia. Luego abordamos dos casos seleccionados como potencialmente innovadores, que se enmarcan en proyectos de extensión universitaria, los resultados obtenidos de ellos y un análisis de ambas experiencias en clave de innovación social. Finalmente, exponemos reflexiones y recomendaciones.

1) Los proyectos de extensión presentan diversas modalidades: Proyectos de Extensión de Interés Social (PEIS); de Interés Institucional (PEII); Acciones de Extensión al Territorio (AET) y Prácticas de Extensión de Educación Experiencial (PEEE). 


\section{Enfoques y perspectivas en los que se inscribe la extensión universitaria}

La extensión posee diversos enfoques que manifiestan la heterogeneidad de perspectivas y tradiciones teóricas de las que se ha ido nutriendo en los distintos momentos de su devenir histórico. Así, es posible reconocer posiciones que la entienden tanto en su altruismo como acción desinteresada hacia los más desfavorecidos-, o en su rol de divulgación de conocimiento (sin énfasis en la retroalimentación que ofrece el territorio), o en la vinculación empresarial (mirada que cobró fuerza en la década de 1990, cuando se instaló el rol de la universidad como satisfactora de necesidades de las empresas en una espiral de beneficios económicos), o bien -y como oposición a la anterior- desde su misión social de creación de conciencia, el despertar de la capacidad de análisis crítico y la acción eficaz sobre la base de una participación política activa y la retroalimentación constante con el medio social de la que ella misma forma parte.

De allí que las prácticas que se implementan en nombre de la extensión manifiestan los paradigmas en los que se sustentan. En este sentido, Agustín Cano Menoni (2017) desarrolla una aproximación a los principales cauces teóricos desde una perspectiva crítica de la extensión. De los cinco que dicho autor presenta, seleccionamos cuatro de ellos, los que a continuación detallamos sintéticamente:

a) La extensión crítica integrada al currículo universitario, como estrategia de integración curricular al proceso de la formación académica.

b) Incubadoras de emprendimientos asociativos y cooperativos populares en el marco de la economía social, lo que requiere de políticas de Estado y de un acompañamiento, por parte de las universidades, que trascienda lógicas de funcionamiento tanto partidarias como asistencialistas.

c) Enfoque territorial, desde programas universitarios integrales basados en el vínculo entre formación, investigación y cooperación comunitaria. Los procesos de intervención están radicados territorialmente, desde una perspectiva interdisciplinaria y con mecanismos de participación y convalidación social.

d) Extensión e "intensión universitaria" articulada a los movimientos sociales. Dicha vinculación recupera la tradición extensionista de universidades populares. Incorpora la agenda y los saberes de dichos movimientos a las agendas académicas. Se trata de una universidad sensible a la pluralidad intelectual, estética y social, que presta atención a los saberes por fuera de las rutinas académicas. En esa misma perspectiva, Tatián remite a la "intensión universitaria":

"Extensa e intensa, la universidad se abre así a una composición con heterogeneidades múltiples en procura de lo común —se abre a una comunidad de los diferentes. ¿Cómo pensar lo común entre la universidad y los movimientos sociales? Y también: ¿qué es lo común entre las distintas ciencias y los diferentes movimientos sociales? Este interrogante no propone el hallazgo de lo que cosas distintas tienen en común, sino una exploración de algo que los diferentes pueden en común. Así, lo común no es lo ya dado de lo que se dispone sino el efecto de una voluntad de encuentro - o de una apertura a la aleatoriedad de los encuentros-, de un trabajo, y una conquista conjunta de acciones y nociones que precipitan una convergencia política; en otros términos: lo común es la conquista de una autotransformación que conmueve las identidades involucradas por la elaboración conjunta de una diferencia y la creación de una novedad". (2013:6) 
Desde las perspectivas mencionadas, cabe preguntarse en el contexto actual cuáles son las prioridades que la extensión debiera replantearse, cómo garantizar la construcción de agendas consensuadas territorialmente, que reflejen las preocupaciones y problemáticas que atraviesan los sectores más vulnerables y que interpelan al mundo académico.

Por otro lado, consideramos que toda acción de extensión implica una intervención en un estado de cosas dado, en un territorio específico y en un entramado de relaciones particulares. En este sentido, la intervención social se entiende como una actividad que se realiza de manera formal u organizada, que responde a necesidades sociales $\mathrm{y}$, específicamente, intenta incidir favorablemente en la interacción de las personas, aspirando a una legitimación pública o social. También conlleva la generación de acontecimientos, el despliegue de estrategias en un contexto histórico, social, político, económico, intentando captar la singularidad, el detalle de las trayectorias de los actores. Por lo tanto, como esquema de acción responde a necesidades sentidas y se considera como la oportunidad para el reconocimiento del otro como sujeto portador de historicidad, de cultura, de lazos.

Reflexionar en torno a la intervención interpela las prácticas, los saberes que se ponen en juego y el registro de mirada desde el cual se conciben. Lleva a develar lo que el contexto y el clima epocal ocultan.

\section{La innovación social en los procesos de intervención}

El concepto de innovación ha tenido una amplia difusión en el marco de las ciencias sociales. En su concepción canónica, estuvo estrictamente ligado a desarrollos científico-tecnológicos, fundamentalmente aplicados a productos provenientes de la industria manufacturera, con una fuerte impronta mercantil. Esta concepción fue ampliándose a principios del siglo XXI; se formularon nuevos interrogantes y se propusieron interpretaciones que, entre otros aspectos, incluyeron la mirada sobre el elenco de agentes potencialmente innovadores así como respecto de los productos y procesos derivados de ellos.

"A la innovación tecnológica originaria, preconizada por la escuela schumpeteriana para incrementar la productividad y competitividad de las empresas, se le han añadido la innovación de servicios, de procesos, de marketing, organizativa, educativa, urbana, científica y más recientemente la innovación social que agrupa diversas actividades innovadoras". (Echeverría, 2006:616)

En el año 2003 el grupo CRISES (Centre de Recherche Sur les Innovations Sociales), de la Universidad de Quebec, en Montreal, publicó documentos que abordan dicho concepto, sus orígenes, evolución, como también su vinculación con las teorías sociológicas del cambio social. Para el CRISES, la innovación social está ligada a los cambios que los actores sociales van generando para satisfacer necesidades, encontrar solución o aprovechar oportunidades para modificar relaciones sociales, transformar un marco de acción o dar lugar a nuevas orientaciones culturales. Otra de las definiciones es la propuesta por la CEPAL, para la cual la innovación en el campo social es resultado de un largo proceso histórico, de un cúmulo de intentos fallidos y pequeñas mejoras que, en un momento crítico, cambian el signo de la tendencia, la dirección de un proceso, la calidad de un producto o la prestación 
de un servicio. Es decir, la innovación remite a cambios que se fueron gestando en el marco de una determinada cultura tecnológica, artística, científica, filosófica u organizativa. Así, la innovación remite a la praxis que genera el cambio y es capaz de sostenerlo en el tiempo y espacio en el que se inscribe.

Por su parte, para Abreu Quintero (2011), la innovación social es un fenómeno complejo y multidimensional que ha pasado en poco tiempo de ser emergente y marginal a ocupar una posición relevante en el discurso político-social. Se han ido tramando diversas miradas y formulando nuevos interrogantes e interpretaciones que incluyen, entre otros aspectos, posicionamientos sobre el elenco de agentes potencialmente innovadores, así como sobre los productos y procesos derivados de ellos. Empero, Hernández Ascanio, Tirado Valencia y Ariza Montes, luego del análisis de 50 definiciones y orientaciones del concepto de innovación social, concluyen que las mismas

"oscilan entre las que plantean que las innovaciones sociales son aquellas que surgen cuando el mercado no puede satisfacer las demandas sociales, y las que se presentan como una definición omnicomprensiva de innovación social, en la que la innovación clásica o de empresa constituye un subconjunto". (2016:178) ${ }^{2}$

Considerando lo anterior, y aun afirmando que el concepto se presenta difuso, ambiguo y hasta muchas veces contradictorio, si se realiza un análisis integral de las distintas cosmovisiones, se recuperan sentidos compartidos respecto de las implicancias del término innovación social. Así, es posible referenciar la misma a procesos de satisfacción de necesidades humanas, a partir de lo cual origina cambios y transformaciones en las relaciones sociales (Estado-mercado-comunidad) con una fuerte impronta de base ciudadana que interpela o pretende interpelar a las agendas y a las políticas públicas.

Por otra parte, la innovación social privilegia la riqueza de los procesos colectivos y remite a dos dimensiones: objetiva y subjetiva. La primera pasa por reformular modelos de gestión que puedan garantizar mecanismos participativos, mayor eficiencia en la respuesta a los problemas sociales, incorporación de nuevas tecnologías, tanto en la resolución de problemas como en relación a la comunicación de los procesos y las prácticas, de modo de potenciar su sustentabilidad, y la posibilidad de replicabilidad en contextos similares. En cuanto a la dimensión subjetiva, donde hay innovación social se generan aprendizajes como consecuencia de los lazos sociales originados, que son los que, en definitiva, otorgan sentido y significación a las prácticas, debido tanto a consensos como a conflictos, en el escenario de la intervención. La dimensión aludida también da cuenta, como sostiene Vaccarezza: "de los procesos de interrelación social que se establecen con otros agentes sociales ajenos al ámbito científico-académico y que se refieren al uso o eventualidad de uso del conocimiento producido" (2009:137). Dichas interacciones se materializan, en parte, en los productos logrados, dejan sus marcas, a modo de tatuajes, de las trayectorias compartidas durante las intervenciones. Atendiendo a esta complejidad, definimos a la innovación social como un proceso que surge en espacios multiactorales a partir de la identificación consensuada de un

2) Un análisis respecto de las vertientes conceptuales de innovación e innovación social puede encontrarse en Beltramino y Theiler (2018). 
problema a escala microsocial, que se transforma en el objeto de intervención sustentado en determinadas tradiciones teóricas. Promueve lazos sociales y aprendizajes que se reflejan en el conjunto de prácticas que los actores intervinientes despliegan, vinculados a trayectorias previas, a condiciones presentes, a posiciones ocupadas dentro del espacio social. No implica una mera transferencia sino un trabajo colaborativo en el cual la participación y el compromiso de los agentes son factores decisivos. Su análisis implica tener siempre como horizonte un aspecto más amplio vinculado al Estado y sus políticas como condiciones para su realización (ver Figura 1).

Figura 1. Innovación social.

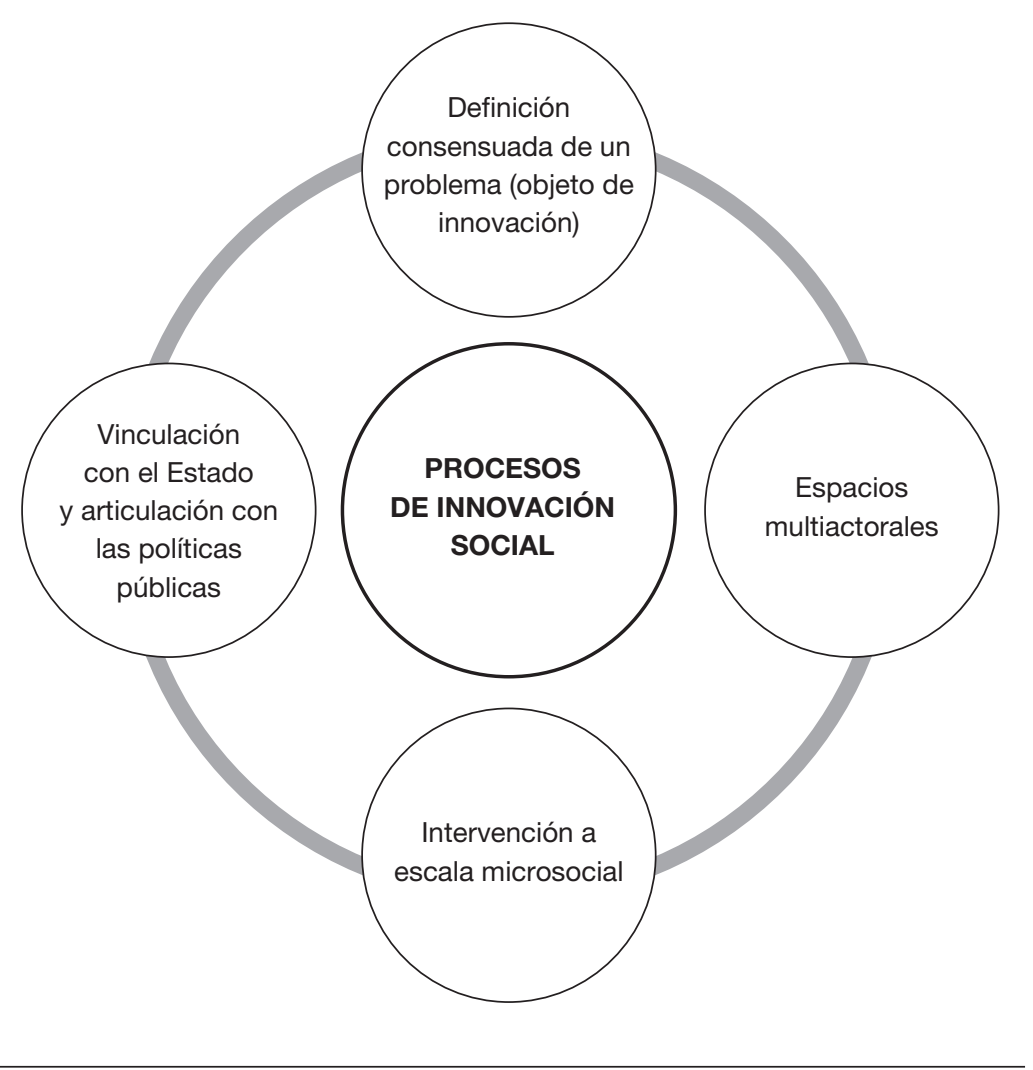

Fuente: elaboración propia.

El análisis de las acciones de extensión universitaria, en clave del concepto de innovación social que acuñamos, atiende a los siguientes criterios que formulamos:

1. El contexto institucional de la intervención a escala microsocial.

2. Espacios multiactorales participativos, como el escenario de las intervenciones.

3. Historicidad de las intervenciones, que remite tanto a las acciones previas a la ejecución de los proyectos como al conocimiento de los equipos intervinientes involucrados en las experiencias de extensión. 
4. Paradigma en el que se sustenta (tradiciones teóricas y las perspectivas desde los modelos de extensión planteados).

5. La situación problemática que dio origen a las intervenciones sociales.

6. Los productos y/o resultados alcanzados, en el marco de los procesos desarrollados.

7. Los aprendizajes, en relación a la circulación de conocimientos, incorporación de información sobre actores, territorios, como de valores vinculados a prácticas colectivas y solidarias.

8. Vinculación con el Estado y articulación con las políticas públicas (asociadas al problema abordado).

Acerca del CAI+D "Análisis de las innovaciones sociales, a partir de la transferencia científico-tecnológica, entre la Universidad Nacional del Litoral e instituciones de la esfera pública"

El objetivo general del CAl+D de referencia consistió en reconocer, describir y analizar las prácticas de innovación social en el marco de los procesos de transferencia de conocimientos científicos-tecnológicos entre la UNL y las instituciones de la esfera pública.

Para ello, el equipo investigador se planteó un conjunto de interrogantes que a continuación exponemos:

- ¿En qué medida la transferencia de conocimientos científico-tecnológicos ha posibilitado la generación de innovaciones en las prácticas institucionales, de carácter tanto endógeno como exógeno? Las endógenas remiten al estilo de gestión y gerenciamiento, que hacen al fortalecimiento organizacional. Las de carácter exógeno dan respuesta a las necesidades sociales y su impacto sobre el contexto de intervención. ¿En qué medida son sustentables y a la vez replicables en situaciones similares?

- ¿Cómo se produce el encuentro entre quienes transfieren conocimientos y técnicas y quiénes son sus destinatarios? ¿Cómo participan en el proceso de transferencia, qué entramado relacional se construye?

- ¿Qué rol juegan los equipos docentes que intervienen, son intermediarios o mediadores en las relaciones de intercambios que se producen? ¿Qué consecuencias generan los intercambios con relación a las prácticas institucionales, a la solución del o los problemas que dieron lugar a la demanda de vinculación/transferencia?

- ¿Qué características ponen en juego los actores? ¿En qué medida se han apropiado de saberes y de conocimientos compartidos durante el proceso de intercambios?

- ¿Cómo se fue generando el diálogo con los agentes externos (como interlocutores, que aportan los recursos técnicos complementarios, la reflexión, el ofrecimiento de una mediación educativa)?

- ¿En qué momento se producen los cambios en las prácticas institucionales como para que signifique un salto cualitativo en las intervenciones?

- ¿Qué recursos y activos se movilizan? ¿Con qué capital social cuentan?

La investigación se focalizó en aquellos proyectos de extensión ya finalizados correspondientes a las convocatorias de los años 2008, 2010 y 2011. Originalmente, fueron relevados 
50 de ellos en sus distintas modalidades: Proyectos de Extensión de Interés Social (PEIS); Proyectos de Extensión de Interés Institucional (PEII) y Acciones de Extensión al Territorio (AET). Para la selección de los potencialmente innovadores se tuvieron en cuenta elementos como: los actores involucrados, los beneficiarios, la finalidad de los proyectos, los objetivos, los logros y los productos generados. Esta información se obtuvo a partir de los informes de avances y finales de cada uno de ellos disponibles en la Secretaría de Extensión Social y Cultural de la UNL. Con posterioridad, el equipo investigador encuestó a los directores de los proyectos y entrevistó a los beneficiarios de los mismos. Desde esa exploración, se construyó una base de datos con 21 proyectos que abarcaron diferentes temáticas (alimentos de interés social, medioambiente, delito, derechos humanos, desarrollo territorial, desarrollo rural, economía, salud, género, niñez, entre otros).

\section{Presentación de los dos casos seleccionados como potencialmente innovadores y resultados de los mismos}

Abordamos aquí dos proyectos de extensión que consideramos potencialmente innovadores ya que responden a varios de los criterios anteriormente mencionados. Cabe remarcar que, si bien las prácticas desde la extensión no están planteadas en términos de innovación social, el poder descifrarlas en dicha clave nos llevó a bucear en las marcas que las mismas fueron dejando en los territorios y en los actores involucrados en los procesos de intervención social.

\section{PEIl: "Construyendo redes de economía social entre la Universidad, organizaciones sociales y el gobierno local" (2010-2012)}

El proyecto tenía como finalidad contribuir al fortalecimiento institucional y a la capacidad de gestión de las organizaciones sociales en su articulación con la UNL y la Municipalidad de Santa Fe. Estuvo dirigido a reflexionar sobre los mecanismos ligados a experiencias en el campo de la Economía Social y Solidaria y a realizar acciones concretas para la promoción y fortalecimiento de las prácticas en el territorio, articulando espacios académicos, actores sociales y políticas públicas. Ello permitió no solo consolidar los procesos de capacitación y acompañamiento sino también afianzar los vínculos institucionales con agentes públicos, en especial con el gobierno local, junto al cual se consensuó un proceso de acompañamiento a Pequeñas Unidades Productivas (PUP) en el marco del programa municipal de promoción de emprendimientos productivos sociales: Programa Puente. ${ }^{3}$ Los emprendedores evaluaron la experiencia como pertinente y valiosa con relación a tres cuestiones: al proceso de formación, a la oportunidad de conocerse y generar vínculos entre ellos y a la necesidad de que estos espacios de aprendizajes puedan sostenerse en el tiempo. ${ }^{4}$

3) El Programa Puente es una propuesta de la Dirección de Trabajo y Empleo del gobierno de la ciudad de Santa Fe. El mismo surgió a fines del año 2009 y está orientado a emprendedores productivos sociales, a través del registro, capacitación, seguimiento y asesoramiento continuo, a quienes además se les brinda posibilidades de comercialización en el espacio público, eventos, ferias, etcétera.

4) En cada uno de los encuentros se destinaba un tiempo para el análisis y reflexión en torno a los contenidos desarrollados, como así también a otros aportes que los emprendedores consideraban significativos. 
Simultáneamente al desarrollo del PEll de referencia, la provincia de Santa Fe se incorporó, a través de la Subsecretaría de Economía Social, en el ámbito del Ministerio de Desarrollo Social, con el objeto de acompañar las experiencias socioproductivas existentes en la ciudad. ${ }^{5}$

En este contexto surgió el Centro de Emprendedores de la Economía Social y Solidaria (CEES), espacio que inicialmente fue gestionado entre la UNL (Secretaría de Extensión), el gobierno de la provincia de Santa Fe, a través del Ministerio de Desarrollo Social, y el municipio local, a través del Programa de Economía Social. Posteriormente, con la transferencia ministerial, fue coordinado por el Ministerio de la Producción. Su creación respondió, entonces, a un conjunto de condiciones y desarrollo de prácticas que favorecieron su materialización, a través de lo cual "los actores involucrados cooperan por una necesidad mutua y comparten los riesgos a fin de alcanzar un objetivo común" (Cardarelli y Rosenfeld, 2002:5).

\section{Descripción de los principales resultados logrados}

Esta integración interinstitucional tuvo como resultado la conformación del Centro de Emprendedores de la Economía Social y Solidaria. Los acuerdos logrados a partir del PEIl mencionado se hicieron efectivos con la celebración de un convenio en el que cada una de las instituciones intervinientes comprometió líneas, programas y proyectos para consolidar los procesos de fortalecimiento de las PUP en Santa Fe:

- La Universidad promovió la creación del Programa de Extensión de Economía Social y Solidaria y la articulación con la cátedra de Economía Social y Solidaria en la Facultad de Ciencias Económicas (FCE), la cual, desde el proceso de formación y articulación académica, vincula a estudiantes y docentes con las acciones desarrolladas desde el CEES. - El gobierno de la provincia de Santa Fe, a través de la Subsecretaría de Economía Social, destinó partidas presupuestarias y redefinió líneas y políticas públicas en virtud de integrarlas a la propuesta; al respecto, se destaca el Fondo Rotatorio de Microcrédito y la campaña "Justo lo que necesito", de promoción del comercio justo y el consumo responsable.

- La Municipalidad de Santa Fe conformó la Dirección de Economía Social y puso a disposición del CEES el Registro de Emprendedores y Productores Locales (REPLo), a la vez que ejecutó la puesta en valor del espacio y avanzó en la coordinación con otras áreas del municipio para otorgar más y mejor visibilidad y dinamismo a las prácticas productivas.

Los antecedentes de las primeras intervenciones permitieron reconocer que la capacitación en técnicas de gestión, administración y comercialización es una etapa requerida pero no suficiente en los procesos de sostenibilidad y apropiación social. Por ello se comprendió la necesidad de abordar propuestas integrales y programáticas desde las cuales acercar los aportes técnicos y metodológicos. Esto motivó la presentación de nuevos proyectos de extensión, docencia e investigación, avanzando en la integración pública y la gestión participativa.

5) Para ampliar la información respecto de los procesos de transformación del gobierno provincial con relación a la economía social, recomendamos consultar Casella (2015). 
Entre los nuevos aportes se destacan:

- Articulación con la Cátedra de Costos y Gestión de la FCE, donde los estudiantes sustituyen un trabajo práctico por la elaboración de la matriz de costos de los productos de los emprendedores. ${ }^{6}$

- Aportes desde la Facultad de Arquitectura, Diseño y Urbanismo (FADU) de la UNL con relación a la estética de los productos.

- Puesta en funcionamiento de una plataforma de comercio virtual a través de un proyecto de investigación, aprobado por el Consejo Interuniversitario Nacional (CIN-CONICET): "Análisis de las iniciativas de Economía Social y Solidaria en la ciudad de Santa Fe. Desarrollo de un Mercado Solidario" (FCE-UNL).

- Desarrollo de un mapeo de iniciativas de economía social en la ciudad a través de un proyecto de investigación orientado a problemáticas sociales y productivas (Cardozo; Serafino; Sotto y Tealdo, 2017).

Hasta el momento, han transitado por este espacio unos 600 emprendedores, se han entregado más de 200 microcréditos (con una tasa de devolución del $95 \%$ ), se han conformado 35 proyectos productivos integrados, se ha elaborado la matriz de costos de más de 50 emprendedores, a la vez que se han dictado más de 40 cursos y talleres de formación y la misma cantidad de instancias de debate y reflexión (conferencias, asambleas, presentaciones de líneas y programas institucionales, entre otras). También se registra la participación de más de 150 estudiantes en diversas acciones académicas.

Al tratarse de un espacio de cogestión, coexisten en tensión distintas cosmovisiones referidas a procesos del campo de la Economía Social así como las disputas de poder, especialmente entre los actores provenientes del ámbito público estatal. Otro factor que incide en el CEES remite a la lógica temporal de las intervenciones, ya que los tiempos de la gestión por parte de las agencias gubernamentales que participan generalmente no acompañan los procesos de apropiación por parte de los actores sociales, en este caso particular, de los emprendedores, con relación a la incorporación de prácticas asociativas y solidarias.

EI CEES constituyó una experiencia novedosa que permitió articular diversas jurisdicciones gubernamentales, la Universidad, a través de las distintas áreas intervinientes y los emprendedores ligados a prácticas socioproductivas.

A causa de las tensiones políticas que se gestaron con el resultado de las elecciones legislativas del año 2017, los vínculos entre los gobiernos local y provincial se fueron debilitando, socavando a su vez al propio Centro de Emprendedores y reflejando la debilidad del espacio en cuanto a la apropiación del mismo por parte del grupo de emprendedores.

\section{PEIS: “Lado B: prácticas artísticas en contextos de encierro" (2011-2013)}

El proyecto tuvo como objetivo general contribuir al fortalecimiento de la condición de sujetos de derecho de las personas privadas de libertad, incrementando sus posibilidades de

6) Puede ampliarse la información en cuanto a los resultados de la educación experiencial en costos y gestión para el Centro de Emprendedores de la Economía Social y Solidaria en el artículo realizado por los propios integrantes de la cátedra: Puccio; Valli; De Greef y Fail (2017). Resultados y desafíos de la educación experiencial en costos y gestión para el Centro de Emprendedores de la Economía Social y Solidaria. Revista +E, 6(6), 438-445. Recuperada de: https://doi.org/10.14409/extension.v1i6.6376 
autonomía, de manera que resultaran menos vulnerables a los procesos de degradación que son característicos de las situaciones de encierro. El mismo fue gestándose en un período de 10 años como resultado de diversas intervenciones realizadas con jóvenes encarcelados, con quienes se habilitaron espacios que permitieron que ellos expresaran sus deseos y ensayaran sus verdades.

La experiencia se inició en el año 2007, cuando un grupo de internos de la Unidad Penal № II, cárcel de Las Flores, participó de un espacio interdisciplinario, con periodicidad semanal, en el que la lectura y la escritura de textos literarios, así como las prácticas artísticas, ocuparon un lugar central. Dichas acciones, que comenzaron a realizarse en el contexto del Proyecto de Educación Universitaria en Prisiones de la UNL, se enmarcaron, a partir de marzo de 2011, en el PEIS de referencia, que promovió el diálogo entre distintas disciplinas y prácticas expresivas, como las artes visuales, el cine, la literatura, el teatro, rastreando puntos de contacto entre las mismas.

El abordaje interdisciplinario contribuyó a ampliar el conocimiento y la experiencia social y cultural de cada sujeto, promovió posibilidades que les ayudaran a "escapar" del encierro, ya fuera mediante la imaginación o la perspectiva de reinserción social que la incursión en el arte abre. En principio, los encuentros realizados comprendían lectura de textos que sus participantes elegían. Luego, estos jóvenes privados de su libertad fueron los protagonistas de las producciones. Uno de ellos compartió, en el marco del taller, una pieza poética de su autoría. ${ }^{7}$

Descripción de los principales resultados logrados

EI PEIS tuvo una fuerte impronta pedagógica al promover, desarrollar y acompañar cuatro grandes propuestas:

1. La revista Lado B Bitácora del Encierro.

2. Adaptación de la historieta El pueblo amurallado a guión cinematográfico.

3. Teaser y rodaje de 17 escenas para el cortometraje de El pueblo amurallado. ${ }^{8}$

4. El programa radial $L a d o B$, en FM Espejo, que transmitía desde dentro de la cárcel de Las Flores.

En las entrevistas efectuadas al equipo extensionista, se hicieron manifiestos los desafíos de la intervención social propios de los obstáculos del entramado de vinculaciones con las

7) “Cuando se tiene una idea clara, se tiene la verdad. Rectángulos, rectángulos y más triángulos, todo cambia... Tristeza que invaden, puñales que se ven, cegados vienen por el hombre y arremeten. Triángulos que caen, esperanzas que matan, sueños que queman. Hilos de fino oro, son ligaduras que atan... Todo se manifiesta. Rectángulos, pobres y angustiados rectángulos. El silencio los acalla. Los perros que ladran del otro lado... se oye el murmullo. Y, de este lado todo, frías noches de invierno, todo silencio, tumbas y tumbas y tumbas... Una lágrima que cae llena de dolor y, a lo lejos, los sollozos de una madre. Sentimientos de ilusión, de ese amor incondicional, que sufre, pero que todo lo puede. Pasan los inviernos, primaveras y veranos, una vez más, no es lo mismo... todo cambia, hasta los puñales que matan. Qué injusticia, es real. Pero quedan los sueños y las esperanzas, que son mías. Esa es la verdad."

8) Con relación a dichas producciones, la codirectora sostuvo: "El pueblo amurallado es la historia de su amigo, en la que dice: 'le extinguieron la luz de los ojos por dos ruedas, por dos pastillas', y agrega: 'yo sujeté la paloma, me la puse al cuello y ahí la deidad apareció', es decir, la paloma es también la soga, porque él se quiso suicidar porque a su amigo el sistema de salud lo dejó morir. Entonces, la idea es que ellos, a través del arte, puedan reflexionar sobre todas estas cuestiones, sobre sus derechos, porque solo conocen sus obligaciones... Esto empodera al sujeto". 
personas encarceladas; la escasa constancia de los participantes en la realización de actividades complejas, su permanencia en el espacio educativo y la procedencia de los mismos, ligada a situaciones de extrema vulnerabilidad y desafiliación social.

Los procesos de aprendizaje, en su mayoría, son atravesados por problemas de comprensión textual, dificultad que se refuerza debido a la escasez de herramientas didácticas, material bibliográfico, tecnología, infraestructura y otros elementos que permitan llevar a cabo proyectos educativos sólidos y permanentes.

Por otra parte, existen dificultades relativas a la "naturalización" en las personas detenidas en cuanto a los abusos o impugnación de los derechos garantizados, y que puede ser explicada, al menos en parte, por el desconocimiento de los derechos y garantías ciudadanas y de los mecanismos para hacerlos efectivos.

La experiencia aludida resultó significativa y valiosa para quienes participaron de ella, fundamentalmente por el proceso construido en el marco de las interacciones generadas entre la comunidad carcelaria, integrantes del equipo extensionista y referentes de agencias de gobierno (Fondo Nacional de las Artes - Secretaría de Derechos Humanos de Santa Fe - Secretaría de Asuntos Penitenciarios y Ministerio de Innovación y Cultura), que aportaron también a la misma. Fueron el marco desde donde se desplegaron prácticas creativas, donde sus beneficiarios fueron descubriendo el poder de las palabras y las cosas.

El proceso se vio interrumpido por cambios en la gestión del instituto carcelario y en el equipo extensionista, que no pudo continuar la intervención debido, además, a múltiples razones (finalización del proyecto, cuestiones laborales y/o personales, entre otras).

\section{Análisis de los procesos de extensión en clave de innovación social}

Párrafos arriba señalábamos que comprender los procesos de intervención social en clave de innovación social implica, inicialmente, dar cuenta de las trayectorias (históricas, sociales, culturales) de las experiencias y reconocer, a su vez, que estas dinámicas se producen en espacios multiactorales complejos donde, y sobre la base de alguna problemática considerada social y colectivamente relevante, se despliegan acciones para dar respuesta. A partir de allí se generan nuevos aprendizajes y transformaciones y se abren nuevas intervenciones que otorgan continuidad a los procesos iniciados.

Lo abordado hasta el momento habilita a plantear que la innovación social no puede ser concebida solamente como resultado. Se trata de entender el propio proceso que permite transformar las dinámicas de relaciones entre los agentes implicados y analizar los aprendizajes, como también la utilidad de los conocimientos que fueron circulando en los espacios generados a partir del entramado vincular entre actores territoriales y los provenientes del mundo académico (ver Cuadro $N^{\circ} 1$ ). 
Cuadro $\mathbf{N}^{\circ}$ 1: Presentación de los dos casos, analizados en clave de innovación social.

\begin{tabular}{|c|c|c|}
\hline $\begin{array}{l}\text { Criterios de análisis de la } \\
\text { innovación social }\end{array}$ & $\begin{array}{l}\text { PEIl "Construyendo redes de economía } \\
\text { social entre la Universidad, organizacio- } \\
\text { nes sociales y el gobierno local" }\end{array}$ & $\begin{array}{l}\text { PEIS “Lado B: prácticas artísticas en } \\
\text { contextos de encierro" }\end{array}$ \\
\hline $\begin{array}{l}\text { 1. Contexto institucional de la } \\
\text { intervención }\end{array}$ & $\begin{array}{l}\text { - Ejido urbano de la ciudad de Santa Fe, donde } \\
\text { se distribuyen distintos emprendimientos pro- } \\
\text { ductivos de subsistencia. }\end{array}$ & - Unidad Penal № II, cárcel de Las Flores. \\
\hline 2. Actores (espacios multiactorales) & $\begin{array}{l}\text { - Emprendedores del campo de la economía } \\
\text { familiar/individual. } \\
\text { - Municipalidad de la Ciudad de Santa Fe. } \\
\text { - Gobierno de la provincia de Santa Fe. } \\
\text { - UNL. }\end{array}$ & $\begin{array}{l}\text { - Jóvenes en contexto de encierro. } \\
\text { - UNL. } \\
\text { - Personal de la Unidad Penal N II, cárcel de } \\
\text { Las Flores. } \\
\text { - Agencias gubernamentales: Secretaría de } \\
\text { Asuntos Penitenciarios de la Provincia de } \\
\text { Santa Fe, Ministerio de Innovación y Cultura } \\
\text { de la Provincia de Santa Fe. } \\
\text { - Fondo Nacional de las Artes - Secretaría de } \\
\text { Derechos Humanos de Santa Fe. }\end{array}$ \\
\hline 3. Historicidad (trayectorias previas) & $\begin{array}{l}\text { - Capacitaciones a emprendedores sociopro- } \\
\text { ductivos a cargo de docentes y especialistas } \\
\text { de la UNL, en el marco del Programa Puente } \\
\text { (2008/2011). }\end{array}$ & $\begin{array}{l}\text { - Prácticas de la UNL en el marco del } \\
\text { "Proyecto de Educación Universitaria en } \\
\text { Prisiones" (2004-2012). }\end{array}$ \\
\hline 4. Paradigma Teórico & $\begin{array}{l}\text { - Economía Social y Solidaria. } \\
\text { - Economía Popular. }\end{array}$ & $\begin{array}{l}\text { - Enfoque de los derechos humanos desde } \\
\text { una perspectiva crítica y liberadora. }\end{array}$ \\
\hline $\begin{array}{l}\text { 5. Situación problemática (objeto de } \\
\text { la intervención) }\end{array}$ & $\begin{array}{l}\text { - Dispersión y desconexión de acciones y } \\
\text { políticas públicas en las PUP en la ciudad de } \\
\text { Santa Fe. } \\
\text { - Escasos niveles de integración entre las PUP } \\
\text { de la ciudad de Santa Fe. }\end{array}$ & $\begin{array}{l}\text { - Representaciones sociales estigmatizantes } \\
\text { sobre las personas en contextos de encierro. } \\
\text { - Baja autoestima de los jóvenes privados de } \\
\text { su libertad, con incidencia en la construcción } \\
\text { identitaria de los mismos y de sus proyectos } \\
\text { de vida. } \\
\text { - Desconocimiento, por parte de las personas } \\
\text { en situación de encierro, de los derechos } \\
\text { ciudadanos y las garantías constitucionales. }\end{array}$ \\
\hline $\begin{array}{l}\text { 6. Productos/Resultados } \\
\text { alcanzados }\end{array}$ & $\begin{array}{l}\text { - Centro de Emprendedores de la Economía } \\
\text { Social. } \\
\text { - Entrega de más de } 200 \text { microcréditos (con } \\
\text { una tasa de devolución del } 95 \% \text { ). } \\
\text { - } 35 \text { proyectos productivos integrados. } \\
\text { - Matriz de costos de más de } 50 \text { emprende- } \\
\text { dores } \\
\text { - Dictado de más de } 40 \text { cursos y talleres de } \\
\text { formación y la misma cantidad de instancias } \\
\text { de debate y reflexión (conferencias, asambleas } \\
\text { y presentaciones de líneas y de programas } \\
\text { institucionales). } \\
\text { - Participación de más de } 150 \text { estudiantes en } \\
\text { diversas acciones académicas. }\end{array}$ & $\begin{array}{l}\text { - Revista Lado B Bitácora del Encierro. } \\
\text { - Adaptación de la historieta El pueblo amu- } \\
\text { rallado a guión cinematográfico. } \\
\text { - Teaser y rodaje de } 17 \text { escenas para el corto- } \\
\text { metraje de El pueblo amurallado. } \\
\text { - El Programa Radial Lado B, en FM Espejo, } \\
\text { que transmitía desde dentro de la cárcel de } \\
\text { Las Flores. }\end{array}$ \\
\hline
\end{tabular}


7. Aprendizajes incorporados

8. Vinculación con el Estado y articulación con las políticas públicas
- Diseño e implementación de modelos de gestión asociada y participativa entre Estado, academia y organizaciones sociales, como mecanismo facilitador de procesos colectivos y potencialmente sustentables.

- Sostenibilidad de las PUP (tanto mercantil como asociativa).

- Fortalecimiento del entramado vincular entre los emprendedores.

- Configuración de espacios que posibilitaron prácticas asociativas y cooperativas.

- Municipalidad de la Ciudad de Santa Fe Programa Puente (2009-2013). Política de promoción del empleo y el autoempleo que lleva adelante la Dirección de Trabajo y Empleo del gobierno de la ciudad de Santa Fe. Creó el Registro de Emprendedores y Productores Locales (Ordenanza 11641/09).

Programa Economía Social (2013-2016). Dirección de Escuelas de Trabajo (Ordenanza 12244). - Gobierno de la Provincia de Santa Fe Subsecretaría de Desarrollo Territorial y Economía Social (2011-2015). Ministerio de Desarrollo Social de la Provincia de Santa Fe: Campaña Justo lo que necesito. Campaña de sensibilización por un consumo responsable. Programa Banco Solidario. Fondo Rotatorio creado por aportes del Ministerio de Desarrollo Social de la Provincia de Santa Fe y ejecutado por la Municipalidad de Santa Fe.

Plan Abre (2013-2015). Eje de intervención: Ideas en Marcha (Porgrama interministerial, el eje que se señala lo ejecuta el Ministerio de Desarrollo Social).

Ministerio de Producción: Productos de mi tierra (programa de marca colectiva, Resolución 449/13).
- El arte como movilizador de procesos creativos, que se materializaron a través de los productos logrados.

- La posibilidad de generar un espacio donde fue posible reconocer derechos, lo cual aportó al fortalecimiento de las identidades y empoderamiento de los sujetos involucrados. - Desarrollo de prácticas colectivas, cuyos resultados fueron significativos, ya que permitió a los jóvenes en situación de encierro, desarrollar recursos creativos, como oportunidad para poder pensarse desde otro lugar.

- Políticas de derechos humanos.

- Programas del Ministerio de Innovación y

Cultura de la provincia de Santa Fe.

- "Hacia una política penitenciaria progresista en la Provincia de Santa Fe. Documento Básico". Secretaría de Asuntos Penitenciarios del Ministerio de Seguridad de la Provincia de Santa Fe (2008). En dicha publicación se expresan como principios orientadores de la política penitenciaria: reducir daños; abrir la prisión a la sociedad; democratizar la prisión; promover y asegurar derechos; reintegrar socialmente a pesar de la prisión.

Fuente: elaboración propia.

El cuadro expone un conjunto de variables que remiten al concepto de innovación social que consensuamos y que compartimos oportunamente en el presente artículo. A partir de las mismas pudimos establecer semejanzas y diferencias entre los casos analizados. Por un lado, el contexto de la intervención refiere al espacio geográfico-institucional desde donde se planifican las intervenciones, que a su vez constituyen respuestas a las problemáticas que fueron abordadas en el marco de los proyectos de extensión seleccionados. En ambos procesos intervinieron una multiplicidad de actores pertenecientes a las esferas pública y privada, como el caso del Centro de Emprendedores, que decidieron incorporar estrategias colectivas a los procesos socioproductivos, las que se han manifiestado en prácticas asociativas y solidarias. Con relación a los jóvenes en contexto de encierro, los dispositivos 
generados en torno a expresiones artísticas posibilitaron procesos lúdicos y aportaron al fortalecimiento de sus identidades y al despliegue creativo de sus capacidades.

Tanto el PEIl como el PEIS abordados remiten a historias y vínculos previos que incidieron en los productos logrados como resultado de distintas intervenciones a las que hace referencia el cuadro. Finalmente, el acompañamiento de las políticas públicas identificadas generó un marco de contención y continuidad a las acciones desarrolladas durante la ejecución de los proyectos.

\section{A modo de reflexión}

Como expresamos al inicio, el presente trabajo fue desarrollado con el objetivo de reflexionar sobre las intervenciones sociales desde la extensión universitaria en clave de innovación social, analizando las relaciones que se generan entre los equipos extensionistas y los actores territoriales. Para ello, indagamos en los dos proyectos de extensión y los resultados alcanzados por los mismos. Si bien las prácticas de extensión universitaria no han sido pensadas en clave de innovación social, analizar dichos procesos desde este enfoque nos permitió profundizar en torno a las marcas que dejaron en los actores involucrados. Estas remiten tanto a la integración de miradas bajo un objetivo común, la incorporación de información del medio en el que estas prácticas se despliegan, como a la generación de una ecología de saberes y el abordaje colectivo de problemáticas consensuadas en espacios multiactorales.

Por otro lado, el análisis de las innovaciones sociales desde los proyectos seleccionados, en su vinculación a programas y/o políticas sociales, implica tener siempre como horizonte un aspecto más amplio que remite al Estado y sus políticas, que desnuda una particular política económica como también una mirada atenta al contexto donde se inscriben.

Otro aspecto a tener en cuenta es la variable temporal. Existen tiempos subjetivos y objetivos, individuales y sociales de construcción de relaciones que han de ser considerados en las políticas de generación o estímulo a la innovación social. Nos referimos específicamente a la existencia de un tiempo cronológico (duración de las intervenciones) y de un tiempo lógico (apropiación social-empoderamiento).

Cabe remarcar que, como debilidad, aparece la limitada sustentabilidad de los procesos, la cual se reitera no solo en los proyectos referenciados sino en la mayoría a los que tuvimos acceso en el marco del proyecto CAl+D que nos posibilitó hacer el análisis compartido.

A modo de cierre, cabe decir que sería recomendable que los proyectos de extensión incorporen en su formulación la mirada de la innovación social como una manera de rescatar los procesos de interacción entre los actores del ámbito académico y los sujetos que transitan los territorios.

Finalmente, consideramos que la universidad se constituye en un actor estratégico, con capacidad para el desarrollo de procesos potencialmente innovadores, para lo cual se requiere, a su vez, de ciertas condiciones contextuales como estructura de oportunidades que propicien dichas prácticas. En este marco, resulta central el acompañamiento del Estado a través de políticas públicas, de la academia en la generación de espacios de diálogo y en las concertaciones multiactorales y de la sociedad civil como actor con capacidad crítica y autonomía de gestión. 


\section{Referencias bibliográficas}

Abreu Quintero, J. (2011). Innovación social, conceptos y etapas. Daena, International Journal of Good Conscience, 6(2), 134-148.

Beltramino, T. y Theiler, J. (2017). Extensión universitaria e innovación social: reflexiones en torno a los vínculos entre la universidad y los actores sociales. En Revista +Eversión en línea, 7(7), 84-96. Santa Fe, Argentina: Ediciones UNL.

Cano Menoni, A. (2017). La extensión universitaria y la Universidad Latinoamericana: hacia un nuevo "orden de anticipación" a 100 años de la revuelta estudiantil de Córdoba. En Revista +E versión en línea, 7(7), 6-23. Santa Fe, Argentina: Ediciones UNL.

Cardarelli, G. y Rosenfeld, M. (2002). La gestión asociada: Una utopía realista. Recuperado de http://www. coodi.com.uy/redoeste/docs/bibkiiogeneral/la\%20gestion\%20asociada\%20Una\%20utopia\%20realista\%20-\%20Cardarelli-Rosenfeld\%202002.pdf (28/08/17).

Cardozo, L.G.; Serafino, E.; Sotto, O. y Tealdo, J. (2017). Mapeo de la Economía Social y Solidaria en el municipio de Santa Fe (Argentina): creación de una base de datos actualizada de las diferentes experiencias. Cooperativismo y Desarrollo, 25(11), 1-20. INDESCO, Instituto de Economía Social y Cooperativismo, Universidad Cooperativa de Colombia. Recuperada de: https://revistas.ucc.edu.co/index.php/co/article/view/1760 Casella, M. (2015). El desafío de construcción de una política de promoción de la Economía Social y Solidaria. El caso del Gobierno de la Provincia de Santa Fe. En Revista +Eversión en línea, 5(5), 142-149. Santa Fe, Argentina: Ediciones UNL.

Echeverría, J. (2006). El Manual de Oslo y la Innovación Social. Departamento de Ciencia, tecnología y Sociedad. Consejo Superior de Investigaciones Científicas, Madrid. Fundación Ikerbasque, Bilbao. Recuperado de arbor. revistas.csic.es/index.php/arbor/article/view/210 (22/06/14).

Fundación CRISES (2003). Las innovaciones sociales. Recuperado de http://crises.uqam.ca/le-centre/presentation.htm|

Gobierno de Santa Fe. Ministerio de Seguridad. Secretaría de Asuntos Penitenciarios (2008). Hacia una política penitenciaria progresista en la Provincia de Santa Fe Documento Básico. Recuperado de http://www. santafe.gov.ar/index.php/web/content/download/56511/274496/file/Descargar.pdf

Gobierno de Santa Fe (2015). Plan de desarrollo. Recuperado de http://www.santafeciudad.gov.ar/gobierno/ plan_desarrollo_santa_2020/programa_gobierno_20112015.html (28/06/17).

Hernández Ascanio, J.; Tirado Valencia, P. y Ariza Montes, A. (2016). El concepto de innovación social: ámbitos, definiciones y alcances teóricos. Revista de Economía Pública, Social y Cooperativa, (88), 165-199. ClRIEC-España.

Kessler, M.E. y otros. Proyecto Curso de Acción para la Investigación y el Desarrollo (CAl+D) "Análisis de las innovaciones sociales, a partir de la transferencia científico-tecnológica, entre la Universidad Nacional del Litoral e instituciones de la esfera pública" (convocatoria 2011).

Lozeco, J.C. (2015). La integración institucional para el desarrollo de la Economía Social y Solidaria. La experiencia del Centro de Emprendedores de la Economía Social en Santa Fe, Argentina. En Revista +E versión en línea 5(5), 174-181. Santa Fe, Argentina: Ediciones UNL.

Tatián, D. (2013). La invención y la herencia Notas liminares para una universidad abierta. Recuperado de http://nupsi.org/wp-content/uploads/2013/08/Diego_TatianInvencion_democratica_de_la_Universidad_San_ Pablo.pdf (22/08/17).

Vaccarezza, L.S. (2009). Las relaciones de utilidad en la investigación social. Revista Mexicana de Sociología [en línea], (71, diciembre-sin mes). Recuperada de: http://www.redalyc.org/articulo.oa?id=32119200006 (16/09/2017). 